\title{
Primary school children's knowledge of, and attitudes towards, healthy computer use
}

\author{
T. Tran ${ }^{\mathrm{a}, \mathrm{b}}$ and M. Ciccarellia, ${ }^{\mathrm{a}, \mathrm{b}}$ \\ ${ }^{a}$ Centre for Research into Disability and Society, School of Occupational Therapy and Social Work, \\ Curtin University, Perth, WA 6845, Australia. \\ ${ }^{\mathrm{b}}$ Curtin Health Innovation Research Institute, Perth, WA 6845, Australia.
}

\begin{abstract}
Background: Australian children as young as five years are using computers in the home and school environments with increased frequency and longer duration as they age. Consequently, they may be exposed to risk factors for the development of physical health problems described in studies of adult computer users. To date schools have focused on providing children with the latest computer technologies; with far less attention given to appropriate workstations, ergonomics education and incorporating safe computing practices. By better understanding children's attitudes and knowledge, a directional approach to the development of an ergonomics education program may be established using a health promotion framework to encourage healthy computing behaviors. Objective: The purpose of this study was to identify children's knowledge of, and attitudes towards healthy computing. Method: A cross-sectional inferential study of 537 Australian school children (aged 9 to 11 years) from Catholic Education Schools across Western Australia was conducted. Multiple logistic regression analyses identified that children's beliefs and attitudes about the negative health effects of computers were found to be most associated with unhealthy computing behaviors. Results: Children lacked sufficient knowledge about the importance of postural and task variation, but had sufficient knowledge about adjusting workstation furniture specific to healthy computer use.
\end{abstract}

Keywords: children, computer ergonomics, knowledge, attitudes, behaviors

\section{Introduction}

\subsection{Background Literature}

Australian children as young as five years of age use computers in home and school environments, with frequency and duration increasing with age [1]. Consequently, children may be exposed to the same risk factors associated with musculoskeletal and visual health complaints described in studies of adult computer users [3]. Educators and the government in Australia have focused on providing school children with access to computer technologies in the learning environment [5]; however, there has been little attention to promoting healthy computing practices at school or home.

Preliminary evidence is available on the subject of children's knowledge about the physical health risks associated with computer use. It is commonly believed that children lack knowledge of issues relating to musculoskeletal stress [23]. Prior studies found that when children experienced discomfort and pain during computer use, they seldom sought medical attention or reported to a parent or teacher $[14,17]$. It may be that they fear their computer access will be restricted or removed as a consequence. It may also be that children cannot associate the discomfort they felt with their awkward sitting postures at school [16].

Furthermore, there is limited evidence available specifically regarding children's attitudes and the physical health risks of computer use. Based on evidence of children's general attitudes towards computers, there is general consensus that children have positive attitudes towards computers. The majority $(67 \%)$ of students in a previous study reported that they felt computers facilitated their learning and many wanted to continue using computers even after finishing their education [21]. This study did not find a clear relationship between discomfort and negative attitudes towards computer use, thus indicating that

\footnotetext{
*Corresponding author. E-mail: M.Ciccarelli@curtin.edu.au
} 
children still held positive attitudes towards computers even when discomfort was experienced during computer use. Other studies report that children express more positive attitudes towards computer use in the home than in their school environments $[10,13]$. This may be due to the perception that computing is more enjoyable at home than at school $[10,13]$. An explanation for this may be that children are able to participate in non-educational games for leisure in the home environment which they may find more enjoyable.

Understanding children's knowledge of, and attitudes towards, healthy computing practices, can assist in the development of ergonomics education programs using a health promotion framework to promote healthy computing among children.

\subsection{Study objectives}

The purpose of this study was to (i) determine children's knowledge about computer ergonomics; and, (ii) determine children's attitudes towards healthy computer practices.

\section{Method}

\subsection{Study sample}

This study aimed to recruit a sample of 600 participants from across Western Australia to achieve a power of 0.80 , alpha level of 0.05 and a minimum effect size of 0.05 . All primary and composite (both primary and secondary) schools $(n=130)$ from the 2008 Directory of Catholic Schools in Western Australia was considered in the sampling process. Using the Socio-Economic Indexes for Areas [2], schools were stratified into four categories: three socioeconomic status (SES) categories (low, medium and high) among metropolitan schools, and one rural category. The homogeneity of participants' characteristics within each school category assists with improving the representativeness of the study sample to the wider community, and reduces sampling error and bias.

This study was approved by the Human Research Ethical Committee at Curtin University and the Director of the Catholic Education Commission of
Western Australia. Informed consent was received from participants and their parents/guardians.

\subsection{Outcomes measure}

The Children's Healthy Computing QuestionnairePrimary/Lower School Version (CHQ-PLS) [9] was used to collect data for this study. The standardised paper- and-pencil questionnaire consisted of 56 items across three subscales: knowledge, attitudes and behaviors. The knowledge items collected nominal data using a multiple choice format. The attitude items collected ordinal data using a Likert scale. A mixture of nominal and ordinal data was collected in the demographic and behavior sections, using mostly a "tick in the box" response format [9].

\section{Results}

\subsection{Participant demographics}

Fourteen schools from the metropolitan area participated in this study. The final sample involved 537 participants (representing a 56\% response rate and $89.5 \%$ of the anticipated sample size). There was an almost even representation of gender in the sample; with 264 males and 269 females. The mean (SD) age of participants was 10.7 (0.47) years, with a range of 10-12 years.

\subsection{Children's knowledge about healthy computing}

A maximum score of 18 is possible in the knowledge section of the outcome measure (CHQ-PLS). The mean (SD) total knowledge score was 12.9 (2.0); range 6-17.

Results for the knowledge items and related constructs are presented in Table 1. Overall, participants scored highest in the 'Monitor' and 'Keyboard and mouse' constructs and lowest in the 'Frequency and duration of physical activity' and 'Duration, variation and breaks' constructs 
Table 1

Results of individual knowledge scores and related constructs

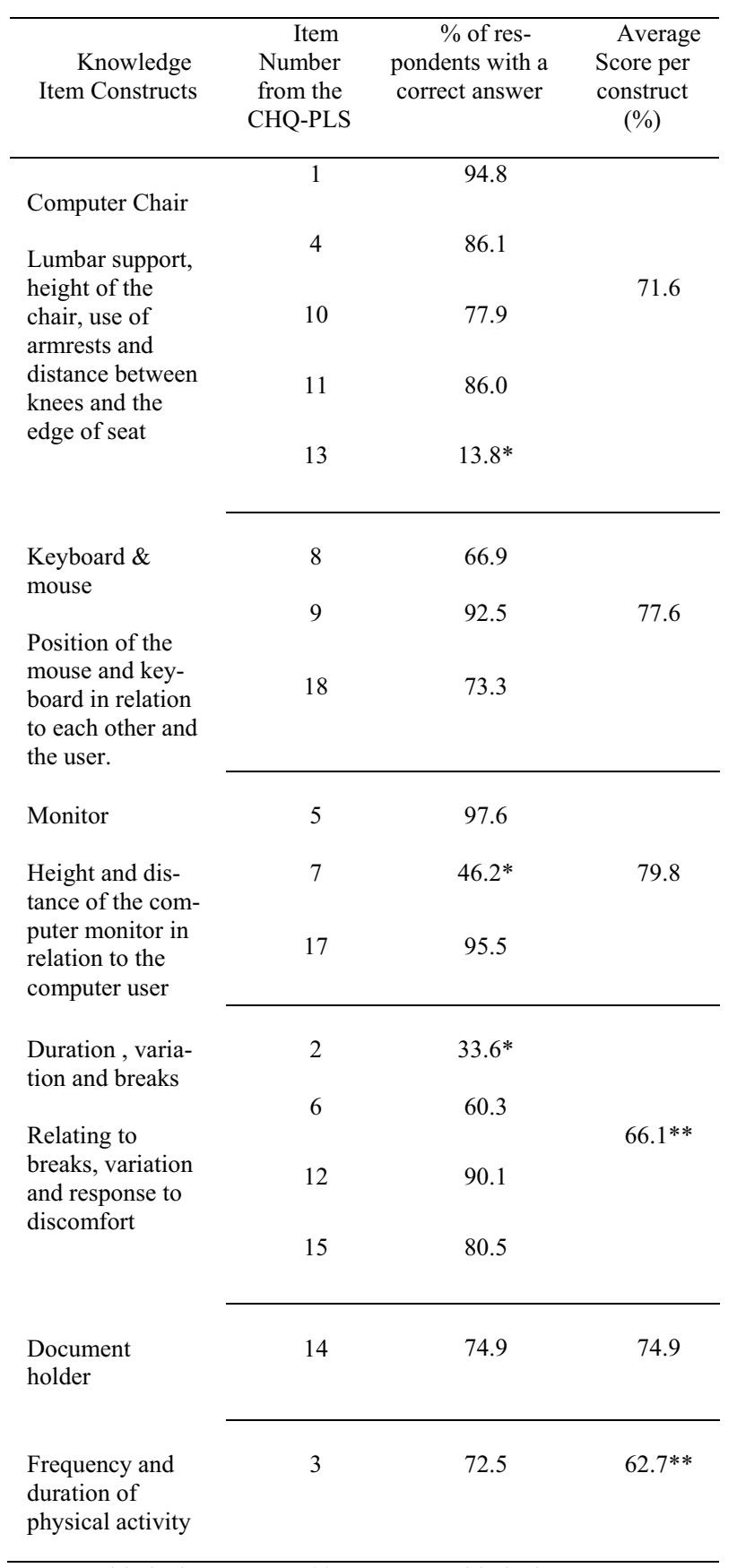

*Item with the lowest score **Constructs with the lowest score

\subsection{Children's attitudes about the effects of computer use on their health}

The forty-three percent of participants agreed that using a computer could cause them to get sore muscles, and sore eyes (72\%). Sixty-two percent of participants agreed with the statement "I would be worried if my muscles or eyes got sore when using a computer"; however the same proportion disagreed with the statement "If I get sore muscles using a computer, I will not be able to join in activities with my friends." In other words, participants were unable to recognize the potential impact that physical health symptoms associated with computing can have on their ability to partake in other daily activities. Seventy-four percent of participants disagreed with the statement "I would not tell anyone if I get soreness or pain in my eyes or muscles when I was using the computer."

Over three-quarters of participants believed they knew what to do to set up their workstation correctly; but $54 \%$ reported "It is difficult to remember to adjust my computer and chair so that my computer posture is healthy". Sixty-eight percent agreed with the statement "If I set up my computer and chair to suit my body before I use it, I can help stop my muscles and eyes becoming sore"; however, most participants believed that it was difficult to develop safe computing habits because of issues related to adjusting the physical environment for optimal computer use.

Male participants were more likely to adopt negative attitudes about the effects of computer use on their health, compared to females. They were less concerned about the onset of discomfort to their muscles or eyes, correct workstation setups and benefits of remembering to take frequent breaks compared to the female participants.

Just over one -third of participants were unsure of whether their parents were concerned about them using computers in a healthy way. However, they also believed that if their parents, teachers and friends encouraged them to adopt healthy computing behaviors, then they would change their behaviors.

\section{Discussion}

\subsection{Children's knowledge about healthy computing}

The participants were most knowledgeable about how to adjust the various hardware components in- 
cluding computer monitor, keyboard and mouse. Although participants had sufficient knowledge of safe ergonomic principles related to workstation set-up, they had difficulty transferring this knowledge into practice. This may have been because most participants reported the furniture they used when using ICT at school was non-adjustable. Inappropriate furniture in classrooms is well documented as a major concern for children's musculoskeletal health [15]

Variation in task, posture and muscle activity is important for the recovery of muscle fibres, thus reducing the potential development of MSDs [3, 8, 12, 20]. Participants in the current study had less knowledge on the principles of variation of posture and tasks. They generally believed that sitting up straight in one position for the entire duration of computer use was best for their health. In contrast, another Australian study found that school children had a basic knowledge of possible risk factors such as awkward postures, long durations and lack of variation associated with discomfort during computer use [4]. The difference in findings between that study and ours may be attributed to the different age groups and sample size between the two studies. The study by Coleman and colleagues [4] involved older participants (aged 11-17 years) compared to the school children in the current study (9-11 years). Furthermore, the findings of this study with its rigorous sampling are more likely to be generalised to other populations, since almost five times more participants were in our study compared to the Coleman et al. (2009) study [4].

Despite about $40 \%$ of the participants in our study reporting they experienced discomfort when using ICT, they lacked knowledge about how to respond appropriately to that discomfort. Other researchers support these results and found that when children experienced discomfort and pain during computer use, they seldom sought medical attention or reported to a parent or teacher $[14,17]$. Thus, they are likely to continue participating in their computer activities despite the onset of discomfort, placing them at an increased risk of possible future computer-related health problems.

Overall, children have knowledge about how to adjust their physical environments to support healthy computing; however, they have less of an understanding of safe computing principles related to variation of posture and tasks. These findings are significant in light of the most current evidence based guidelines that suggests the following recommendations: (i) encourage children to take an active break from the computer every 30 to 60 minutes; (ii) encourage postural variation, and (iii) teach children to respond to discomfort [22]. Incorporating these guidelines into future ergonomic interventions programs to enhance school children's knowledge of safe computing practices are recommend for future research.

\subsection{Children's attitudes about the effects of computer use on their health}

No prior evidence regarding children's attitudes and physical health risks of computer use was available. This study is the first to thoroughly investigate these constructs. Overall, the participants believed that there may be some negative health effects associated with their computer use, and all continued to be frequent users of various technologies.

Children reported that they were not confident to adjust their workstation where they use a computer at school. This suggests that although they had sufficient knowledge regarding this concept, they were not confident in transferring this knowledge into practice in classrooms with a physical layout or furniture that did not match their anthropometric needs or the demands of the computing task.

This study identified that males were less concerned about computers affecting their health, compared to females. Within a health promotion framework, males are more likely to adopt healthy computing behaviors if they have the knowledge, attitudes and skills [7]. There is a need and an opportunity to provide school children with ergonomics education and target changing their beliefs and values to promote better physical health outcomes. Straker (2001) agrees that school children are still likely to be positive about using computers, despite experiencing physical discomfort [21]. In our study, of the $40 \%$ of children reporting ICT-related discomfort, males were less concerned about these symptoms, placing them at greater risk than females in potentially developing physical health problems such as MSDs and visual problems. The participants overwhelmingly disagreed that they would not report their computer related discomfort, which is in contrast to the findings of others $[14,17]$.

Participants from lower SES groups were less concerned about the potential effects of ICT use on their health, when compared to those from higher SES groups. Other researchers have found similar results regarding the relationship between SES and health outcomes. One study involving 754 children (mean age of 9.8 years) and 1142 adolescents (mean age of 14 years) across seven European countries [18], 
found that parents with higher educational status had a positive impact on their children's physical health and well-being, compared to those with lower educational status. These findings suggest that parents of participants from the higher SES categories may be more vigilant and have more knowledge about potential risk factors impacting on their children's health. School children from lower SES backgrounds may therefore, be at greater risk of adopting unhealthy computing behaviors due to less parental guidance.

\subsection{Influence of parents, peers and teachers on children's healthy computing behaviors}

The children's perceptions that they did not know if their parents or teachers were concerned about their health when using ICT suggest a lack of communication between school children and their parents, peers and teachers regarding healthy computing. Thus, a window of opportunity is available to educate and embed positive attitudes in parents, peers and teachers to encourage school children's healthy computer use.

Parents play a critical role in guiding their children's behavior outside the school environment. The degree of concern they have regarding the potential negative impact that computers may have on their children's health and the way they communicate this to their children, are important in shaping children's computing habits. Other researchers found that parents generally perceive computer technology as having a positive influence on their children's lives [26]. Prior research found that parents are concerned about their children's psychological health and safety regarding exposure to inappropriate commercial, sexual and violent content via computers [19]. If parents have less concern about possible physical health risks associated with computer use, it may be because of their lack of awareness of the prevalence of computer related discomfort among children. Parent education on strategies to promote healthy use of computers within a health promotion framework is required, to encourage positive attitudes towards the potential health effects of ICT on school children's physical health.

Participants also believed that their peers influenced their computing habits. There is agreement from others researchers that peers have a very powerful influence on children's attitudes and behaviors [24]. These findings are significant for future ergonomic interventions promoting healthy computing behavior in schools. The environment created in class may be positive or negative, influencing school children's behavior when using computers. Using health promotional principles and participatory ergonomics, it may be beneficial to have a whole class or whole school approach, where peer support is encouraged to promote healthy computing habits.

Teachers' knowledge of and their attitudes towards the potential negative health effects of ICT use among school children are important. If teachers are unaware of the possible negative health problems associated with computer use, then it is likely that similar beliefs are transferred to their students. Other researchers have identified concepts that support these views. A large study of primary school teachers identified that $81.5 \%$ of respondents were not satisfied with their current knowledge of ergonomics, and that $90 \%$ believed it would be beneficial to learn these concepts [6]. Another study involving 218 American teachers found that only $12 \%$ had received information about computer ergonomics [27]. Teachers from that study identified that they would like to learn about: (i) general computer ergonomic guidelines for students, (ii) what are considered to be good work habits, (iii) how to include computer ergonomics in typing classes, (iv) how to adapt available workstation furniture to fit all sizes of students, and (v) how to teach good seated postures and address visual issues. If teachers are open to learning about computer ergonomics, it may be beneficial to introduce these concepts into their tertiary education curriculum [11].

\section{Conclusion}

Children nowadays are exposed to rapidly evolving ICT in both the home and school environments. The use of computers in education, leisure or socialisation occupations have become central for many school children. Evidence suggests that the physical demands of extensive computer use could lead to a wide range of adverse health effects including computer-related musculoskeletal complaints and visual problems.

This study provides valuable information about what primary school children know about healthy computing and their beliefs and attitudes about the health effects of computer use. The findings of this research challenge educators to consider the introduction of ergonomics education to reduce potential risks for computer-related musculoskeletal complaints in children. 
This study identified that school children had insufficient knowledge about the importance of variation of posture and tasks when using computers and that forty percent of participants reported soreness or pain associated with computer use.

Parents and teachers do not appear to engage school children in discussions about healthy computing practices, which may reflect their own lack of knowledge about computer ergonomics. In light of these findings, there is a need to focus on macroergonomics in future interventions to not only instill knowledge about safe computing habits, but also develop strategies to design classrooms that promote positive attitudes towards healthy computing behaviors and empower school children to self-manage their own physical health when using ICT.

\section{References}

[1] Australian Bureau of Statistics, 4901.0 - Children's participation in cultural and leisure activities, (2006), Canberra.

[2] Australian Bureau of Statistics, 2033.0.55.001 - Socioeconomic indexes for areas (SEIFA), Canberra.

[3] M.Ciccarelli, L. Straker, S. Mathiassen and C.Pollock, ITKids Part II : Variation of postures and muscle activity in children using different information and communication technologies, Work 38 (2011), 413-427.

[4] J. Coleman, L. Straker and M. Ciccarelli, Why do children think they get discomfort related to daily activities? Work 32 ( 2009), 267-274.

[5] Department of Education and Workplace Relations, The Digital Education Revolution- putting computers in front of secondary school students, 2009, http://www.deewr.gov.au

[6] S. Dockrell, E. Fallon, M. Kelly, B. Masterson, and N. Shields, School children's use of computers and teachers' education in computer ergonomics, Ergonomics 50 (2007), 1657-1667.

[7] G. Egger, R. Spark and R. Donovan, Health promotion strategies and methods (2nd ed.) North Ryde, N.S.W, 2005.

[8] G. Evans and K. Patterson, Predictors of neck and shoulder pain in non-secretarial computer users, International Journal of Industrial Ergonomics, 26 (2000), 357-365.

[9] M. Hatfield, The development of a measure to assess primary school children's knowledge, attitudes and behaviours relating to healthy computer use. Honours dissertation. Curtin University, 2009.

[10]T. Levine and S. Donitsa-Schmidt, Computer use, confidence, attitudes and knowledge: A causal analysis, Computers in Human Behaviour 14 (1998), 125-146.

[11] S.J. Legg and K. Jacobs, Ergonomics for schools, Work 31 (2008), 489-493.

[12] S. Mathiassen, Diversity and variation in biomechanical exposure: What is it, and why would we like to know?, Applied Ergonomics, 37 (2006), 419-427.

[13]S. Mumtaz, Children's enjoyment and perception of computer use in the home and the school, Computer and Education 36 (2001), 347-362.

[14] S. Murphy, P. Buckle and D. Stubbs, Classroom posture and self-reported back and neck pain in schoolchildren, Applied Ergonomics 35 (2004), 113-120.
[15]S. Oates, G. Evans and A. Hedge, An anthropometric and postural risk assessment of children's school computer work environments, Computers in the Schools 14 (1998), 55-63.

[16]G. Panagiotopoulou, K. Christoulas, A. Papanckolaou and K. Mandroukas, Classroom furniture dimensions and anthropometric measures in primary school, Applied Ergonomics 35 (2004), 121-128.

[17] L.B. Royster, A healthy approach to classroom computers: Preventing a generation of students from developing repetitive strain injuries, North Carolina Law Review 80 (2002), 275314.

[18]U. Rueden, A. Gosch, L. Rajmil, C. Bisegger and U. RavensSieberer, Socioeconomic determinants of health related quality of life in childhood and adolescence: Results from a European study, Journal of Epidemiology and Community Health 60 (2006), 130-135.

[19] M. Shields and R. Behrman, Children and computer technology: Analysis and recommendations, The Future of Children 10 (2000), 4-30.

[20]J.K. Sluiter, K.M. Rest and M.H.W. Frings-Dresen, M. H.W., Criteria document for evaluating the work-relatedness of upper-extremity musculoskeletal disorders, Scandinavian Journal of Work, Environment and Health 27 (2001), 1-102.

[21]L. Straker, Are children at more risk of developing musculoskeletal disorders from working with computers or with paper? Paper presented at the 15 th Annual Conference of the International Society for Occupational Ergonomics and Safety, 2001, Fairfax, Virginia.

[22] L.M. Straker, B. Maslen, R. Burgess-Limerick, P. Johnson and J. Dennerlein, Evidence-based guidelines for the wise use of computers by children: Physical development guidelines, Ergonomics 53 (2010), 458-477.

[23] L.M Straker, B. Maslen, R. Burgess-Limerick and C. Pollock, Children have less variable postures and muscle activities when using new electronic information technology compared with old paper-based information technology, Journal of Electromyography and Kinesiology 19 (2009), 132- 143.

[24]L.M. Straker and C.M. Pollock, Optimizing the interaction of children with information and communication technologies, Ergonomics 48 (2005), 506-521.

[25]L.M. Straker, C.M. Pollock and B. Maslen, Principles for the wise use of computers by children, Ergonomics 52 (2009), 1386-1401.

[26]K. Subrahmanyam and P. Greenfield, Online communication and adolescent relationships, The Future of Children 18 (2008), 119-146.

[27]I. Williams, T. Cook and T. Zigler, Computer Ergonomics for teachers and students. Paper presented at the 14th Triennial Congress of the International Ergonomics Association and 44th Annual Meeting of the Human Factors and Ergonomics Society, 2000, Santa Monica, CA. 